\title{
Geant4 simulations of STIX Caliste-SO detector's response to solar $\mathrm{X}$-ray radiation
}

\author{
Jaromir Barylak ${ }^{1}$, Aleksandra Barylak ${ }^{1}$, Tomasz Mrozek ${ }^{1,2}$, Marek \\ Steślicki $^{1}$, Piotr Podgórski ${ }^{1}$ and Henryka Netzel ${ }^{2}$ \\ ${ }^{1}$ Space Research Centre, Polish Academy of Sciences, ul. Kopernika 11, 51-622 Wrocław, \\ Poland, email: jbarylak@cbk.pan.wroc.pl \\ ${ }^{2}$ Astronomical Institute, University of Wrocław, ul. Kopernika 11, 51-622 Wrocław, Poland
}

\begin{abstract}
Spectrometer/Telescope for Imaging X-rays (STIX) is a part of Solar Orbiter (SO) science payload. SO will be launched in October 2018, and after three years of cruise phase, it will reach orbit with perihelion distance of $0.3 \mathrm{a}$ a. STIX is a Fourier imager equipped with pairs of grids that comprise the flare hard X-ray tomograph. Similar imager types were already used in the past (eq. RHESSI, Yohkoh/HXT), but STIX will incorporate Moiré modulation and a new type of pixelized detectors with CdTe sensor. We developed a method of modeling these detectors' response matrix (DRM) using the Geant4 simulations of X-ray photons interactions with CdTe crystals. Taking into account known detector effects (Fano noise, hole tailing etc.) we modeled the resulting spectra with high accuracy. Comparison of Caliste-SO laboratory measurements of ${ }^{241} \mathrm{Am}$ decay spectrum with our results shows a very good agreement. The modeling based on the Geant4 simulations significantly improves our understanding of detector response to X-ray photons. Developed methodology gives opportunity for detailed simulation of whole instrument response with complicated geometry and secondary radiation from cosmic ray particles taken into account. Moreover, we are developing the Geant4 simulations of aging effects which decrease detector's performance.
\end{abstract}

Keywords. Sun: corona, instrumentation: detectors, methods: numerical

\section{Introduction}

The main objective of the SO, first M-class mission of ESA's Cosmic Vision 2015-2025 programme, is investigation of the connection between the Sun and the heliosphere. SO orbit will be heliocentric with perihelion equal 0.28 AU. Simultaneous in-situ measurements, remote high-resolution imaging and spectroscopic observations of the Sun will be performed with a broad suite of instruments. One of them is the STIX which provide us with images and spectra of the Sun in $4-150 \mathrm{keV}$ range with high spatial and spectral resolutions (Benz et al. 2012).

STIX consists of three main building blocks:

- X-ray window, which provide thermal shielding and rejection of low-energy X-ray photons,

- Imager - 30 pairs of grids with different pitches and orientations which provide Fourier components of solar X-ray emission sources distribution,

- Detector Electronics Module - set of 32 Caliste-SO detectors with on-board computer.

Caliste-SO consist of a CdTe sensor and a dedicated front-end electronics manufactured in 3D Plus technology (Meuris et al. 2014). The crystal sensor has a $100 \mathrm{~mm}^{2}$ area and thickness of $1 \mathrm{~mm}$, and is divided into 12 pixels, which are grouped into four stripes. This arrange allows to detect Moiré pattern shape, which is produced by pairs of grids located 
before each detector, with high accuracy . Moreover, pixels allow to limit too high photon flux observed during large solar flares by disabling some of them thereby reduce active area. Additionally, entire crystal is surrounded by guard ring that eliminates edge effects. There are two electrodes in the CdTe sensor. The entrance electrode (cathode) is made of $15 \mathrm{~nm}$ thick platinum layer. On the opposite side, the multilayer anode, consisting of $50 \mathrm{~nm}$ thick aluminium, $15 \mathrm{~nm}$ titanium and $80 \mathrm{~nm}$ gold layers, is placed.

We performed simulations of Caliste-SO detectors using Geant4 package (Agostinelli et al. 2003) which is a toolkit for simulation of particle interaction with matter. This package is useful for simple simulations as well as analysis of whole large experiments performed e.g. in the Large Hadron Collider. The Geant4 toolkit collects knowledge about Monte Carlo simulations and physical process, and is used in many fields of science, like: nuclear and particle physics, accelerators, space engineering, and medical physics. The package contains a broad suite of physical models (including electromagnetic, hadronic and optical), which covers a wide range of energies from around $250 \mathrm{eV}$ up to several $\mathrm{TeV}$. Implemented physical models are continuously improved and extended.

\section{Detector effects}

During a simulations additionally to photon-crystal interactions the detector effects have to be taken into account. Photons absorbed in a detector crystal generate a electronhole pairs. Next, carriers are transported to electrodes where total charge is counted. Several effects may influence the signal read out on electrodes:

(a) Hole tailing. Holes are characterized by low mobility and life time due to impurities and defects present in crystal. Therefore, not all of them reach electrode which lowers counted total charge and produce a low energy tail for each measured spectral feature. Hole tailing depends strongly on photon absorption depth. Therefore, the tail will be longer for higher energy photons. Hole tailing is described by Hecht equation:

$$
\eta(x)=\frac{\lambda_{h}}{D}\left(1-e^{-\frac{x}{\lambda_{h}}}\right)+\frac{\lambda_{e}}{D}\left(1-e^{-\frac{D-x}{\lambda_{e}}}\right)
$$

where: $\lambda_{e, h}$ - mean free path of holes (h), and electrons (e), D - crystal thickness, $\mathrm{x}-$ photon absorption depth.

(b) Fano noise. Even if absorbed photons have exactly the same energy they create different number of carriers, which produce a broadening of measured spectral features. The FWHM of this broadening is equal $1 / \sqrt{\frac{F \cdot w}{E}}$, where $\mathrm{F}$ is Fano coefficient, $\mathrm{w}$ is mean energy of electron-hole pair, and $\mathrm{E}$ is energy of absorbed photon.

(c) Electronic noise. The result is broadening of spectral features, but it does not depend on photon energy.

(d) Damage layer. It is observed in Caliste-SO sensor front part. Photons absorbed at depth lower than $5 \mu \mathrm{m}$ produce less signal than expected. The result is similar to hole tailing, but it depends on absorption depth, rather than photon energy.

\section{Simulations' results}

We considered CdTe sensor with electrodes divided into pixels (Meuris et al. 2012). Simulations were performed assuming monoenergetic photon source with energy changing from $4 \mathrm{keV}$ to $180 \mathrm{keV}$ in $0.1 \mathrm{keV}$ steps. The photon source was planar with a size equal to a size of CdTe sensor. Photons felt at right angle to the crystal surface and their distribution was uniform. For each energy we simulated 1 million of photons. We 

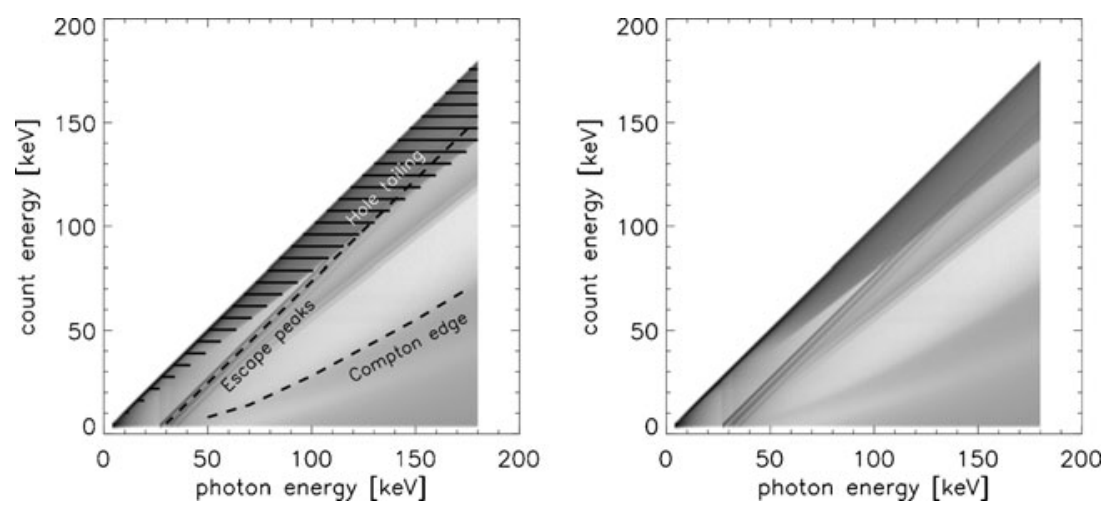

Figure 1. Left panel: DRM of Caliste SO with marked hole tailing, escape peaks, and Compton edge. Right panel: DRM from Geant4 simulations without marked features.

used Livermore physics list, which is dedicated to low energy physics. For photons following effects were taken into account: photoelectric effect, compton scattering, gamma conversion, rayleigh scattering. For electrons we included multiple scattering, Coulomb scattering, ionisation, and bremsstrahlung. Simulated spectrum was compared with laboratory measurements of ${ }^{241} \mathrm{Am}$ performed with real Caliste-SO. The radioactive data from LBNL Isotopes Project database has been used. We have found good, at a level of few percent, agreement between simulations and measurements.

Simulations performed enabled us to calculate a detector response matrix (DRM) for Caliste-SO. Most of the response is in the diagonal elements of the matrix (the blue line). The non-diagonal response contains: escape peaks (four lines parallel to diagonal marked by dotted line), hole tailing (red triangle beneath diagonal), Compton scattering visible below edge marked by dashed line.

Developed methodology give an opportunity for investigation of detector degradation effects and their influence on scientific data analysis and interpretation. Assuming that detector's ageing mainly affects carriers lifetime we were able to simulate response of aged Caliste-SO. For 1\% of carriers original lifetime, which we expect after several years of instrument operation, significant change in detector's response can be observed in a off-diagonal values mainly. Assuming various levels of detectors' degradation we will be able to construct a 3-D DRM which will improve accuracy of data reduction and analysis.

\section{Acknowledgements}

We acknowledge financial support from the Polish National Science Centre grant number 2011/01/M/ST9/06096.

\section{References}

Agostinelli, S., Allison, J., \& Amako, K., et al. 2003, Nucl. Instrum. Meth. A., 506, 250

Barylak, J., Podgórski, P., Mrozek, T., Barylak, A.; Steślicki, M., Sylwester, J., \& Ścisłowski, D. 2014, Proc. of SPIE, 9290, 929037

Benz, A.,O., Krucker, S., \& Hurford, G. J., et al. 2012, Proc. of SPIE, 8443, 84433L

Meuris, A., Hurford, G. J., \& Bednarzik, M., et al. 2012, Nucl. Instrum. Meth. A., 695, 288

Meuris, A., Limousin, O., \& Gevin, O., et al. 2014, Proc. of SPIE, 9154, 91540Y 